\title{
LEEM investigation of the faceting of the Pt covered W(111) surface
}

\author{
K. Pelhos ${ }^{1}$, J.B. Hannon ${ }^{2}$, G.L. Kellogg ${ }^{2}$, T.E. Madey ${ }^{1 *}$ \\ ${ }^{1}$ Department of Physics and Astronomy and Laboratory for Surface Modification \\ Rutgers, The State University of New Jersey \\ ${ }^{2}$ Sandia National Laboratories, Albuquerque, New Mexico 87185-1413
}

\begin{abstract}
$\underline{\text { Abstract }}$
A low energy electron microscope (LEEM) has been used to investigate the faceting of $\mathrm{W}(111)$ as induced by $\mathrm{Pt}$. The atomically rough $\mathrm{W}(111)$ surface, when fully covered with a monolayer film of $\mathrm{Pt}$ and annealed to temperatures higher than $\sim 750 \mathrm{~K}$, experiences a significant morphological restructuring: the initially planar surface undergoes a faceting transition and forms three-sided pyramids with $\{211\}$ faces. The experiments demonstrate the capability of LEEM for imaging both the fully and partially faceted surface. In addition, we have observed the formation of the facets in real time, when $\mathrm{Pt}$ is dosed onto the heated surface. We find that the transition from planar surface, to partially faceted surface, and to fully faceted surface proceeds through the nucleation and growth of spatially separated faceted regions.
\end{abstract}

\footnotetext{
"Author for correspondence, e-mail: madey@physics.rutgers.edu
} 


\section{DISCLAIMER}

This report was prepared as an account of work sponsored by an agency of the United States Government. Neither the United States Government nor any agency thereof, nor any of their employees, make any warranty, express or implied, or assumes any legal liability or responsibility for the accuracy, completeness, or usefulness of any information, apparatus, product, or process disclosed, or represents that its use would not infringe privately owned rights. Reference herein to any specific commercial product, process, or service by trade name, trademark, manufacturer, or otherwise does not necessarily constitute or imply its endorsement, recommendation, or favoring by the United States Government or any agency thereof. The views and opinions of authors expressed herein do not necessarily state or reflect those of the United States Government or any agency thereof. 


\section{DISCLAIMER}

Portions of this document may be illegible in electronic image products. Images are produced from the best available original document. 


\section{Introduction}

Model bimetallic catalysts consisting of thin metal films deposited onto planar single crystal metal surfaces are of great interest to surface scientists due to their interesting physical and chemical characteristics. We have focused recently on studying a special class of bimetallic systems: films up to a few atomic layers thick on the atomically rough (111) surface of bcc metals, such as W and Mo. A special emphasis is placed on the morphological stability of these systems, as certain overlayer films are found to induce a large scale restructuring of the substrate under certain conditions, forming nanoscale facets. Specifically, W(111) and Mo(111) surfaces are found to be morphologically unstable when covered by ultrathin films of $\mathrm{Pt}, \mathrm{Pd}, \mathrm{Au}, \mathrm{Rh}$ or Ir; pyramidal facets are formed when the film covered surface is annealed to temperatures above $-750 \mathrm{~K}[1-3]$. The sides of the pyramids have mainly $\{211\}$ orientations as evidenced by both low energy electron diffraction (LEED) and scanning tunneling microscopy (STM). STM also shows that depending on annealing temperatures and times these facets can grow as large as $\sim 100 \AA$ (in the case of Pd) up to $\sim 1000 \AA$ (in the case of Pt).

There are scientific issues, however, that are yet to be addressed in connection with metal overlayer induced faceting of $\mathrm{W}$ and $\mathrm{Mo}(111)$. These include the nature of facet nucleation, coexistence of faceted and planar surfaces, facet growth (with annealing time), and a high temperature reversible transition from faceted to planar surface[4]. In the present work we use a low energy electron microscope[5] (LEEM) to provide new insights into the $\mathrm{Pt}$ induced faceting of $\mathrm{W}(111)$. Our main objective is to conduct real time "dynamic" observations of the nucleation and growth of Pt induced faceting of W(111).

The main advantage of LEEM is that real time observation of such structural-

morphological changes is possible, with $\sim 70 \AA$ spatial resolution. The low energy electrons that are used to image the surface are diffracted, essentially forming a LEED pattern inside the imaging column of the LEEM. One has a choice between imaging the 
LEED pattern and forming a real image of the surface using any or all of the LEED beams. For a surface that has multiple domains of different surface structures, it is possible to distinguish one domain type from the others when imaging the surface by using a selected LEED spot from that domain structure to form a real space image of the surface. Two of the many excellent examples of this technique are the alternating $2 \times 1$ and $1 \times 2$ reconstructions on neighboring steps of $\operatorname{Si}(100)[6-12]$, and the oxygen induced reconstruction of $\mathrm{W}(100)$ with $5 \times 1$ and $1 \times 5$ domains[13].

The LEED pattern from a faceted bcc(111) surface is a superposition of three distinct bcc(211) patterns. It is possible, therefore, to image the surface through a selected LEED spot belonging to only one of the three facet orientations, and observe individual facets on the surface, provided that the facet size exceeds the resolution limit of the LEEM. Furthermore, under certain conditions a coexistence of faceted and planar regions has been observed using LEED and STM for the Pt/W(111)[14], Pd/Mo(111) and $\mathrm{Au} / \mathrm{Mo}(111)$ systems[4]. With LEEM it is possible to observe the spatial distribution of such coexistence phases. Last, but not least, LEEM is capable of providing a real time view of the transitions from planar to faceted systems, or planar to coexistence to faceted systems, and vice versa. We observe in these experiments the nucleation and growth of faceted domains as the heated surface is dosed with $\mathrm{Pt}$.

\section{Experiment}

The experiments were performed at Sandia National Laboratories using a commercial LEEM developed by E. Bauer[5]. The LEEM apparatus is housed in a conventional stainless steel ultra-high vacuum (UHV) system with an average base pressure of $2 \times 10^{-10}$ torr. The chamber is equipped with a Pt evaporation source for dosing the W(111) sample in-situ, allowing the observation of the surface while it is being dosed. The sample may also be heated in-situ to temperatures up to $\sim 1500 \mathrm{~K}$, by electron bombardment from the back side. Also available are a $\mathrm{K}$ source and an external $\mathrm{Hg}$ arc lamp to perform 
photoemission electron microscopy (PEEM), necessary for the proper alignment of the microscope and the sample. A quadrupole mass spectrometer (QMS) may be used for residual gas analysis.

The LEEM image is formed on a phosphor screen and, using a CCD camera, recorded onto a video cassette. A video image-grabbing software also enables the storage of LEEM pictures on a computer for further analysis.

Attached to the main LEEM chamber are two separate preparation chambers: one for sample cleaning, and the other equipped with a cylindrical mirror analyzer and a concentric electron gun for Auger electron spectroscopy (AES). We use AES to check sample cleanliness and to determine the approximate Pt coverage, using the calibration of $\mathrm{Pt}$ to $\mathrm{W}$ Auger peak ratio versus coverage of previous works $[14,15]$.

The sample is a $\mathrm{W}$ single crystal disk (approx. $1 \mathrm{~mm}$ thick and $1 \mathrm{~cm}$ in diameter) oriented and mirror-polished to within $0.5^{\circ}$ of the (111) plane. A molybdenum sample holder assembly provides for high temperature anneals (up to $2400 \mathrm{~K}$ ), by using a $\mathrm{W}$ filament mounted directly behind the sample for electron bombardment heating, where the sample is kept at a high positive potential (up to 800 Volts) and is heated by electrons emitted as the filament is heated resistively to incandescence; at maximum temperatures the emission current reaches $150 \mathrm{~mA}$. The sample temperature is measured using both an infrared pyrometer and a matched filament pyrometer.

Ultrathin Pt films are deposited from a commercial metal evaporator, in which a Mo rod holding a small Pt ball is heated by electron bombardment. The dosing rate is automatically kept constant by monitoring the beam flux. The maximum flux rate at the surface is approximately $1 \times 10^{14}$ atoms $/ \mathrm{cm}^{2} / \mathrm{min}$, which would result in a W(111) surface fully covered by Pt in approximately 15 minutes. One major problem of the evaporation setup is that the sample is partially covered by a Mo cap, as part of the sample holder assembly, in order to achieve more uniform electric fields above the surface. Furthermore, the Pt doser is mounted at an angle of $\sim 80^{\circ}$ off the sample surface normal. 
The large angle of incidence of the Pt flux results in a further reduction of the dosed surface area, due to shielding by the edges of the Mo cap. Therefore only the central area of the sample $(\sim 15-20 \%)$ is dosed directly. The significance of this arrangement is discussed below.

\section{Results and Discussion}

Previous work has shown that Pt induces faceting of the W(111) surface under certain conditions[14]. A phase diagram has been mapped out (figure 1) as a function of $\mathrm{Pt}$ coverage and annealing temperature, based on LEED observations (note that the phase diagram has been updated and the coverage scale rescaled from that in reference [14] based on more recent experiments[15]). A LEED pattern characteristic of a fully faceted surface is observed if the Pt coverage exceeds a critical coverage of one physical monolayer (i.e. every W surface atom is covered by a Pt atom, $\sim 1.7 \times 10^{15}$ atoms $/ \mathrm{cm}^{2}$ ) and the sample is annealed between 750 and $1200 \mathrm{~K}$ for 3 minutes. At coverages between 0.7 and 1 physical monolayer, or if the annealing temperature is between 1200 and $1300 \mathrm{~K}$, LEED shows a combination of a faceted pattern and a planar W(111) pattern. STM experiments have confirmed the coexistence of a planar region with faceted structures[14, 16]. It has also been shown using STM that the final facet size increases with increasing annealing temperature, from an average of $\sim 150 \AA$ at $880 \mathrm{~K}$ up to $\sim 700 \AA$ at $1400 \mathrm{~K}[14$, 16].

In view of these earlier results, we hare attempted three different sets of experiments to examine the nucleation and growth of faceted regions. First we produced fully or partially faceted surfaces with large facet sizes, expecting to see individual facets that

exceed in size the resolution limit of our microscope (which we estimate to be $\sim 200 \AA$ for the conditions in this experiment). Second, we have dosed Pt in-situ onto a hot $(\sim 1050 \mathrm{~K})$ surface while observing the system with LEEM, therefore attempting to see a "dynamic" horizontal (i.e. constant temperature) cross section of our phase diagram: a transition 
from planar to faceted surface. Finally, we dosed onto a cold surface an amount of $\mathrm{Pt}$ sufficient to induce faceting, and gradually increased the surface temperature beyond the faceting threshold, which gives a "dynamic" vertical (i.e. constant coverage) cross section of the phase diagram. Each of these experiments is discussed below.

\section{III.a. LEED observations}

We have used the LEED capability of the microscope to observe faceting and establish proper dosage levels and annealing temperatures. Since a direct measurement of Pt coverage is not possible to carry out in the LEEM system, we have relied on results of previous work[14], in particular on the phase diagram of figure 1. As mentioned earlier, due to a Mo cap covering the sample, only $15-20 \%$ of the surface is dosed with Pt. Since the overlayer film diffuses to cover the entire surface under the Mo cap upon high temperature annealing, we have to evaporate several (5-7) physical monolayers of Pt onto this small central area to maintain a fully covered surface at elevated temperature, for faceting to be observable.

The identification of faceting of a W(111) surface using conventional LEED has been well described before[17]. The three sided pyramids of the faceted $\mathrm{W}(111)$ surface have equivalent $\{211\}$ faces: (211), (121) and (112). Therefore, the LEED pattern of the faceted W(111) surface is a superposition of three distinct $W\{211\}$ LEED patterns, each rotated $39^{\circ}$ off the surface normal, and rotated $120^{\circ}$ with respect to each other about the surface normal. While LEED beams of the W(111) surface converge onto the specular $(0,0)$ beam normal to the surface as the electron energy is increased, LEED spots from the $\{211\}$ facets will converge onto their respective specular beams, $39^{\circ}$ off the surface normal, near the edge of the LEED screen. By varying the energy of the incident electrons and observing the movement of LEED beams, both fully and partially faceted systems are easily identified.

When using the LEED capability of a LEEM, however, the pattern is slightly different (figure 2). LEED beams from the planar W(111) surface do not move as the 
electron energy is changed[18,19], whereas spots from the $\{211\}$ facets do. In particular, three $(0,1)$ spots from the three different $\{211\}$ orientations (identified on figure 2 ) move symmetrically across the screen as the incident energy is varied; at $\sim 8 \mathrm{eV}$ incident energy they coincide in the center of the pattern. If the pattern is of a partially faceted surface, they also coincide with the $(0,0)$ beam of the planar surface (cf. Figure 3); the importance of this fact in identifying facets on real space LEEM images is discussed in III.b.

Our LEED results are in agreement with previous work[14], although the coverage scale of the phase diagram of figure 1 has been rescaled based on more recent experiments[15]. The clean surface produces a well defined $1 \times 1$ LEED pattern, and using various Pt coverages and annealing temperatures several examples of both partially and fully faceted surfaces have been produced. In addition, the high temperature region on figure 1, identified as "superstructure", has been determined to be $(2 \sqrt{3} \times 2 \sqrt{3}) \mathrm{R} 30^{\circ}$.

Of particular interest is the experiment where the initially clean sample is kept at $\sim 1050 \mathrm{~K}$, and observed with LEED while being dosed with Pt. After 60 minutes of deposition extra beams from $\{211\}$ facets appear on top of the planar $1 \times 1$ pattern, indicating a coexistence of planar W(111) features with $\{211\}$ facets. Upon further Pt dosing (after 90 minutes) beams from the planar $1 \times 1$ pattern disappear, leaving a fully faceted LEED pattern behind. This is in good qualitative agreement with a "horizontal" (i.e. constant temperature) cross section of the phase diagram on figure 1. The quantitative agreement between 60 vs. 90 minutes and 0.7 vs. 1 physical monolayers is also satisfactory, considering the error involved in exact coverage determinations. The LEEM study of this constant temperature cross section is discussed below, in III.c.

\section{III.b. "Static" LEEM observations}

There are two different methods that can be used to generate LEEM images of a partially faceted surface. One may select - using a small aperture - a LEED beam from the planar surface, such as the $(0,0)$ beam, to form an image of the surface. This is referred to as a "bright field image", since the planar surface appears bright on the screen, 
while facets or faceted regions (as well as contaminated areas that do not have the structure of the planar W(111) surface) appear dark (figure 4a).

The bright field mode has the advantage that the incident electron energy may be varied at will, since LEED spots from the planar surface remain stationary. This fact may be used to prove whether the features observed with LEEM are indeed due to facets or faceted regions, using the observation that faceted spots coincide with the planar $(0,0)$ beam at an incident energy of $\sim 7.8 \mathrm{eV}$. Figure 3 shows a series of LEED images from the partially faceted surface at incident energies from $5.6 \mathrm{eV}$ to $12 \mathrm{eV}$, and clearly demonstrates the merging of the facet beams with the $(0,0)$ beam of the planar surface at $\sim 7.8 \mathrm{eV}$. Figure 4 shows a series of bright field images at incident energies from $5.6 \mathrm{eV}$ (figure 4a) to $12 \mathrm{eV}$ (figure 4e). Some of the dark spots visible on figure 4a "light up", as the energy is increased from $5.6 \mathrm{eV}$ to $7.8 \mathrm{eV}$ in figure $4 \mathrm{c}$, then become dark again as the energy is further increased to $12 \mathrm{eV}$ (figure 4e); these initially dark areas therefore are positively identified as being due to faceted regions. The spots that remain dark at $7.8 \mathrm{eV}$ are probably due to surface contamination or defects.

Previous STM experiments performed on the Pt/W(111) system indicate that the average facet size is expected to be approximately $700 \AA$ for this temperature $(1500 \mathrm{~K})$, with fairly narrow size distribution[14, 16, 20]. We conclude, therefore, that the dark areas, approximately 2000 to $3000 \AA$ in diameter, do not correspond to individual facets, but rather to small and fully faceted regions of the surface.

Note that faceted regions appear as bright spots when facet LEED beams coincide with the planar $(0,0)$ beam, rather than the entire screen having a uniform brightness. This might be due to differences in the summed LEED beam intensity from the faceted region, in comparison with that from the planar region. In addition, increased field and hence anomalous focusing effect at the apex and edges of pyramids may further enhance the apparent brightness of the faceted regions. 
The second method of forming LEEM images is to form an image by selecting one of the LEED beams originating from one of the three $\{211\}$ facets - this is referred to as "dark field image". Figure 5a is a dark field image of the fully faceted surface approximately $9 \mu \mathrm{m}\left(9 \times 10^{4} \AA\right)$ across. The sample was prepared by annealing a Pt dosed sample to $-1200 \mathrm{~K}$. According to STM experiments $[16,20]$, facet sizes at this temperature range from $200 \AA$ to $700 \AA$. In this case only one side of each pyramid appears bright, while the dark areas include facets of the other two $\{211\}$ orientations, as well as facets that are smaller than the $200 \AA$ resolution limit. The latter explains why less than one third of the total surface area is bright $(\sim 15 \%)$, although according to LEED there are no planar regions (these would also appear dark) on this surface.

Figure $5 \mathrm{~b}$ is a dark field image of a partially faceted surface approximately $5 \mu \mathrm{m}$ $\left(5 \times 10^{4} \AA\right)$ across. The Pt dosed surface has been annealed to $1400 \mathrm{~K}$. LEED shows a coexistence of planar and faceted patterns, and previous STM experiments show individual pyramids as large as $\sim 1500 \AA$ with extended planar regions $[16,20]$. The few bright spots on figure $5 \mathrm{~b}$ with rectangular symmetry are probably due to such large facets, comparable to facets in atomic resolution STM images of the faceted $\mathrm{Pd} / \mathrm{W}(111)$ system[3].

One might argue that small facets are not visible on LEEM because they are smaller than the transfer width of the low energy electron beam. Note, however, that a fully faceted surface with individual facet sizes that prove to be much too small to be observable by LEEM produce a perfectly well defined LEED pattern. This indicates that the facet size exceeds the transfer width of the electron beam, and the limiting constraint in imaging small facets is the lateral resolution of the microscope. Indeed, fully faceted surfaces for which the maximum facet size is below $\sim 200 \AA$ will appear uniformly gray.

\section{III.c. "Dynamic" LEEM — constant temperature faceting}

The most important advantage of LEEM is its capability of imaging a surface in real time, even surfaces at high temperatures, or surfaces as they are being dosed. The main 
objective of this project was to conduct real time, or "dynamic" observations of the nucleation and growth of Pt induced faceting of W(111).

One set of experiments was aimed at observing the onset of faceting along a horizontal cross section of the phase diagram of figure 1: that is, observing the surface that is kept at a constant temperature as $\mathrm{Pt}$ is being deposited at a slow rate, $\sim 1$ physical monolayer per 15 minutes at the center of the sample. Figure 6 shows a sequence of bright field images from one such experiment, where the sample temperature was kept at $1050 \mathrm{~K}$. The first signs of faint dark spots (indicating facets) appear after $\sim 50$ minutes. After $\sim 60$ minutes well defined small islands of faceted regions are clearly observable (figure 6 a). After $\sim 90$ minutes the surface appears uniformly dark, i.e. fully faceted (figure 6f).

The time delay of $\sim 50$ minutes until the first signs of faceting (rather than $\sim 10-15$ minutes expected from the dosing rate) is due to the fact that at $1050 \mathrm{~K}$ the $\mathrm{Pt}$ readily diffuses over the W(111) surface; Pt may even diffuse to areas under the Mo cap that are not being dosed directly with Pt. Note that desorption of Pt from the W(111) surface does not begin below $\sim 1600 \mathrm{~K}[14]$. The spreading of the Pt film and faceting are two competing processes, until enough $\mathrm{Pt}$ is dosed to completely cover the sample everywhere with at least a one physical monolayer thick film. In fact, if we continue the experiment above, keeping the sample at the same temperature $(1050 \mathrm{~K})$ after the surface is fully faceted, but stop the deposition of $\mathrm{Pt}$, the surface reverts to completely planar form in $\sim 15$ minutes due to the spreading of the Pt film to the point where the critical thickness requirement for faceting is no longer met. If at this point we open the Pt doser while keeping the same sample temperature, the surface completely becomes faceted again in less than $\sim 30$ minutes.

The most remarkable result of the above experiment is that the fully faceted surface is achieved through a nucleation and subsequent growth of a relatively few faceted regions. One possible explanation for our observation is that the Pt film itself grows via this 
nucleation/growth process. Note that the critical film thickness for complete faceting is one physical monolayer $\left(\sim 1.7 \times 10^{15}\right.$ atoms $\left./ \mathrm{cm}^{2}\right)$, which is equivalent to three monolayers using conventional definition. Just before the first signs of faceting become visible, the surface appears to be covered with $2 / 3$ physical monolayers ( 2 geometrical monolayers) of Pt. As the third geometrical monolayer nucleates, and thus the film thickness reaches critical coverage, faceting occurs. The numbers ( $2 / 3$ versus 1 physical monolayer, 60 minutes versus 90 minutes of dosing) are certainly in good agreement with the phase diagram of figure 1.

The processes of film growth during deposition of atoms on surfaces, including the nucleation, growth and coalescence of adsorbate islands, are among the best studied phenomena in surface science. Experimental methods range from diffraction techniques (such as SPA-LEED[21, 22] and thermal energy helium-atom scattering[22-24]) to real space observations using STM[25-30], transmission electron microscopy (TEM) and LEEM[31]; theoretical predictions and models also abound[32-36].

Metal adsorbates, when deposited onto metal surfaces at temperatures $\geq 300 \mathrm{~K}$, often have high mobility and condense into islands[27,28]. These islands are nucleated either at terrace edges or other surface defects such as screw dislocations (heterogeneous nucleation) $[27,31]$, or simply in the middle of wide terraces, if the adsorbate density is high enough for the spontaneous creation of nuclei where the number of atoms exceeds a critical value (homogenous nucleation) $[28,30]$, or both[25, 29].

In our experiments of $\mathrm{Pt}$ on W(111), we believe the growth proceeds in a layer-by layer fashion up to the completion of the first physical monolayer. However, as mentioned above, one physical monolayer includes three geometrical monolayers, where each geometrical monolayer includes adsorbate atoms of the same type of nearestneighbor environment. Our observation indicates that as $\mathrm{Pt}$ is being deposited onto the substrate, the first two geometrical monolayers are completed first; then follows the completion of the third geometrical monolayer via nucleation and subsequent growth of 
islands. This suggests that while $\mathrm{Pt}$ adatoms in the first two monolayers remain relatively immobile on the $\mathrm{W}(111)$ surface, the Pt atoms deposited on top of this surface have higher mobility, and readily diffuse around until sticking onto an already growing island.

It is important to note that as the faceted regions are growing, there are no new regions being nucleated on the remaining planar surface (figure 6). This may be explained by the rapid diffusion of Pt atoms at the elevated temperature of $1050 \mathrm{~K}$, and the very low impingement flux. The combination of the two results in a low probability of island nucleation as compared to the growth rate of existing islands. In addition, $\mathrm{Pt}$ adatoms diffusing on the surface may stick preferentially to already faceted regions.

Note that the complicated interplay between the faceting process and the diffusion of Pt away from the central region is the reason why the idea of a detailed quantitative analysis or a theoretical model has not been attempted for the present data. To obtain data for such analysis, a better control of experimental conditions is necessary, including an improved sample holder design.

\section{III.d. "Dynamic" LEEM — constant coverage faceting}

Another interesting question is, how does faceting occur when the surface is fully covered with Pt beyond the critical coverage, and the sample is heated slowly above the critical temperature of $\sim 750 \mathrm{~K}$ ? Does the whole surface facet at once, or is there a nucleation and growth type process, much like the above case?

Repeated attempts to observe any type of nucleation and growth process using LEEM for such "constant coverage" faceting have failed, in part, for technical reasons. Although LEED indicated that the sample becomes fully faceted upon annealing, we failed to observe the transition with LEEM - due to relatively low contrast and automatic exposure compensation, a simple bright-to-dark transition is very difficult to observe.

The apparent lack of a nucleation and growth type of process (or any other kind of coexistence phase) in a constant coverage faceting experiment is in agreement with previous results. The phase diagram of figure 1 from earlier work[14] does not show a 
coexistence phase at the low temperature boundary of the faceted region - although in those experiments temporal evolution studies were not attempted, the sample was annealed at each temperature for three minutes. Also, detailed STM studies of the initial stages of faceting at constant coverages were conducted for the $\mathrm{Pd} / \mathrm{W}(111)$ system[37], and the surface was found to undergo a uniform roughening/microfaceting, with facet sizes as small as $\sim 20 \AA$.

\section{Conclusions}

We have demonstrated that LEEM is an extremely useful complement to other microscopic and diffraction techniques used to characterize metal film induced faceting of atomically rough bcc(111) surfaces. During Pt deposition onto a heated W(111) substrate we have observed real-time LEEM images of the faceting process, which demonstrate that facets nucleate and grow in spatially separate regions of the surface. The faceted regions grow to cover the entire surface when the Pt coverage reaches $\sim 1 M L$. LEEM also provides a very convenient and immediate comparison of real space images and LEED observations, a particularly useful feature when studying faceting.

The resolution of LEEM does not match that of an STM; however, its convenience and the advantages mentioned above make it very desirable both for identifying new candidates for faceting and for studying the dynamic details of such large scale morphological transformations. In any case, improved sample holder and evaporator designs are both needed for a more thorough and more complete study of the faceting process.

Future LEEM studies of the adsorbate-induced faceting of atomically rough metal surfaces will involve other overlayer metals and possibly other atomically rough substrates (such as an $f c c(210)$ metal surface). Furthermore, there have been literature reports of reversible phase transitions in the case of $\mathrm{Pd} / \mathrm{Mo}(111), \mathrm{Au} / \mathrm{W}(111)$ and $\mathrm{O} / \mathrm{Mo}(111)[4,20]$, where the surface changes from a fully faceted to a coexisting 
planar/faceted surface structure as the temperature increases and reverts to fully faceted as the temperature decreases. Such phenomena clearly call for a detailed LEEM investigation.

\section{Acknowledgment}

This work has been supported, in part, by the US Department of Energy, Office of Basic Energy Sciences. This work was performed at Sandia National Laboratories, Albuquerque, NM. Sandia is a multiprogram laboratory operated by Sandia Corporation, a Lockheed Martin Company, for the United States Department of Energy under Contract No. DE-AC04-94AL8500.

\section{$\underline{\text { References }}$}

[1] J. Guan, R.A. Campbell and T.E. Madey, Surf.Sci. 341 (1995) 311

[2] T.E. Madey, J. Guan, C.-H. Nien, C.-Z. Dong, H.-S. Tao and R.A. Campbell, Surf.Rev.Lett. 3 (1996) 1315

[3] C.-H. Nien and T.E. Madey, Surf.Sci. 380 (1997) L527

[4] K.-J. Song, J.-C. Lin, M.-Y. Lai and Y.-L. Wang, Surf.Sci. 327 (1995) 17

[5] E. Bauer, Rep.Prog.Phys. 57 (1994) 895

[6] M. Mundschau, E. Bauer, W. Telieps and W. Swiech, Surf.Sci. 223 (1989) 413

[7] E. Bauer, M. Mundschau, W. Swiech and W. Telieps, J.Vac.Sci.Technol.A 9 (1991) 1007

[8] W. Swiech and E. Bauer, Surf.Sci. 255 (1991) 219

[9] N.C. Bartelt, R.M.Tromp and E.D. Williams, Phys.Rev.Lett. 73 (1994) 1656

[10] N.C. Bartelt and R.M. Tromp, Phys.Rev.B 54 (1996) 11731

[11] R.M. Tromp and M.C. Reuter, Phys.Rev.Lett. 68 (1992) 820

[12] J.B. Hannon, N.C. Bartelt, B.S. Swartzentruber, J.C. Hamilton and G.L. Kellogg, Phys.Rev.Lett. 79 (1997) 4226

[13] M.S. Altman and E. Bauer, Surf.Sci. 347 (1996) 265 
[14] C.Z. Dong, S.M. Shivaprasad, K.-J. Song and T.E. Madey, J.Chem.Phys. 99 (1993) 9172

[15] K. Pelhos and T.E. Madey, to be published

[16] T.E. Madey, K.-J. Song, C.-Z. Dong and R.A. Demmin, Surf.Sci. 247 (1991) 175

[17] K.-J. Song, R.A. Demmin, C. Dong, E. Garfunkel amd T.E. madey, Surf. Sci. 227 (1990) L79

[18] E. Bauer, Ultramicroscopy 17 (1985) 51

[19] E. Bauer and Telieps, Scanning Microscopy Supplement I (1987) 99

[20] K.-J. Song, C.-Z. Dong and T.E. Madey, in The Structure of Surfaces III, S. Y. Tong, M. A. V. Hove, K. Takayanagi and X. D. Xie (Springer-Verlag: Berlin. 1991) p. 378.

[21] P. Bedrossian, Surf.Sci. 334 (1995) 1

[22] P. Bedrossian, B. Poelsema, G. Rosenfeld, L.C. Jorritsma, N.N. Lipkin and G. Comsa, Surf.Sci. 334 (1995) 1

[23] C. Tölkes, P. Zeppenfeld, M.A. Krzyzowski, R. David and G. Comsa, Phys.Rev.B 55 (1997) 13932

[24] G. Vidali, Surf.Rev.Lett. 4 (1997) 709

[25] S. Horch, P. Zeppenfeld and G. Comsa, Surf.Sci. 331-333 (1995) 908

[26] P.S. Weiss and D.M. Eigler, Phys.Rev.Lett. 69 (1992) 2240

[27] D.D. Chambliss, K.E. Johnson, R.J. Wilson and S. Chiang, Journal of Magnetism and Magnetic Materials 121 (1993) 1

[28] R.Q. Hwang, J. Schröder, C. Günther and R.J. Behm, Phys.Rev.Lett. 67 (1991) 3279

[29] S. Horch, P. Zeppenfeld and G. Comsa, Applied Physics A 60 (1995) 147

[30] J.A. Meyer, P. Schmid and R.J. Behm, Phys.Rev.Lett. 74 (1995) 3864

[31] M. Mundschau, E. Bauer, W. Telieps and W. Swiech, Surf.Sci. 213 (1989) 381 
[32] J.B. Hannon, M.C. Bartelt, N.C. Bartelt and G.L. Kellogg, Surf. Rev. Lett. (1998) to be published.

[33] M. Breeman, T. Michely and G. Comsa, Surf.Sci.Let. 370 (1997) L193

[34] Y. Li and A.E. DePristo, Surf.Sci. 351 (1996) 189

[35] M.C. Bartelt and J.W. Evans, Surf.Sci. 298 (1993) 421

[36] V.P. Zhdanov, Surf.Sci. 392 (1997) 185

[37] C.-H. Nien and T.E. Madey, to be published. 


\section{Figure captions}

figure 1. a) Phase diagram to demonstrate the temperature and coverage dependence for faceting of W(111) caused by thin Pt films, based on LEED observations. Adopted from reference [14], with coverage scale adjusted according to more recent experiments[15].

b) Top and side view of the W(111) surface demonstrates that atoms from the three outermost layers are exposed to the surface - these three atomic layers together form one physical monolayer.

figure 2. LEED image of partially faceted $\mathrm{W}(111)$ using LEEM at $17 \mathrm{eV}$ incident electron energy. Beams from the planar (111) surface are identified as well as beams from the three different facet orientations: (211), (121) and (112).

figures 3 and 4. LEED and bright field LEEM image sequence for a partially faceted surface, with varying incident electron energy: $5.5 \mathrm{eV}, 6.5 \mathrm{eV}, 8 \mathrm{eV}, 9 \mathrm{eV}$ and $12 \mathrm{eV}$ for images a through e, respectively. The coincidence of the $\{211)$ facet LEED beams with the $(0,0)$ beam of the planar surface at $\sim 8 \mathrm{eV}$ corresponds to the brightening of faceted regions on LEEM. (Image size is $\sim 9 \mu \mathrm{m}$ in figure 4.)

figure 5. Dark field image of fully faceted surface (5a; image size $\sim 9 \mu \mathrm{m})$ and partially faceted surface $(5 \mathrm{~b}$; image size $\sim 5 \mu \mathrm{m}$ ). Bright spots are believed to correspond to individual facets.

figure 6. Bright filed image of nucleation and growth of faceted regions on $\mathrm{Pt} / \mathrm{W}(111)$ at constant temperature $(\sim 1050 \mathrm{~K})$ and constant Pt flux. The dosing times are $63,69,75,81,87$ and 93 minutes for images $6 a$ through $6 f$, respectively. Image size is $5 \mu \mathrm{m}$. 

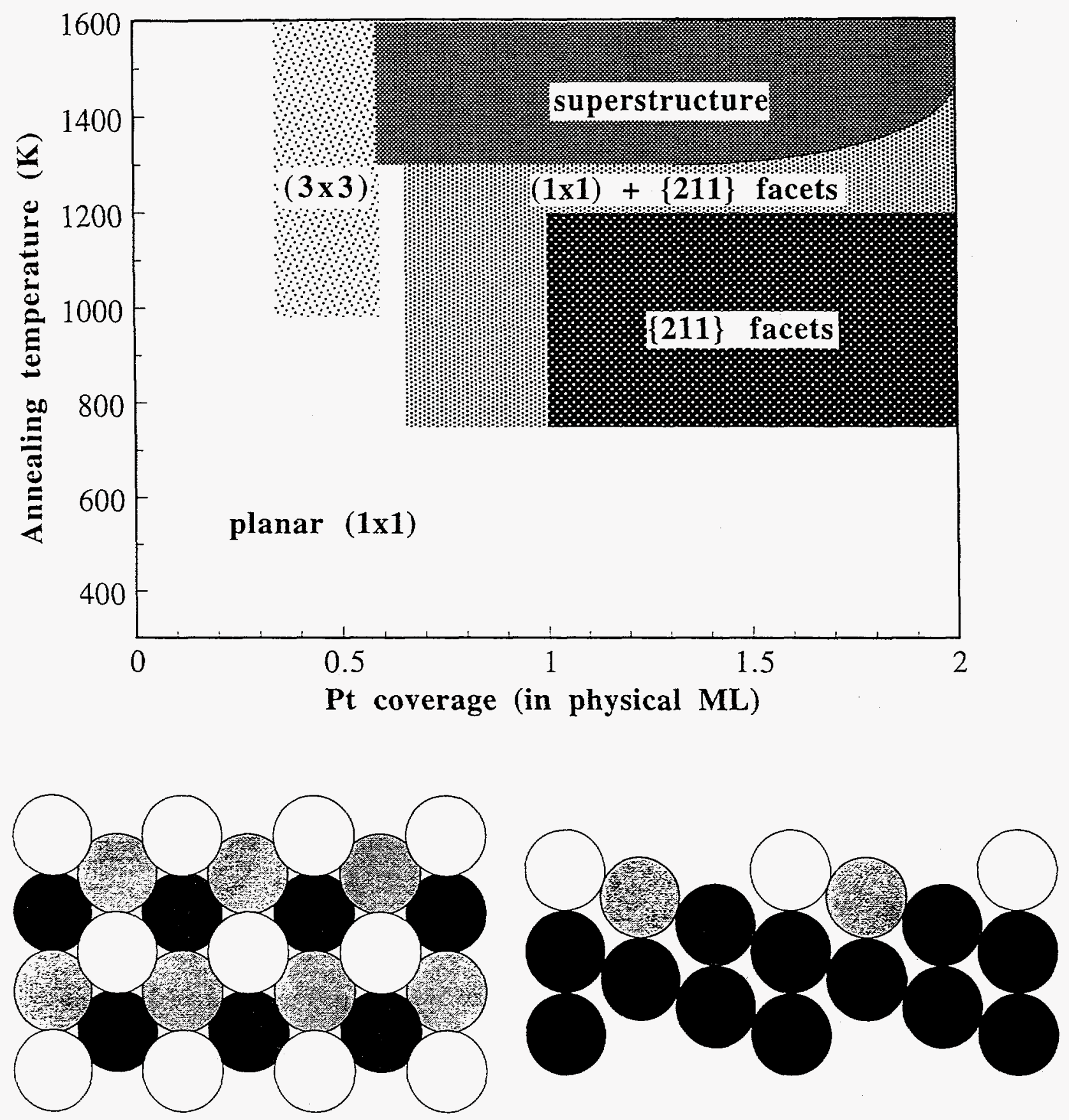

Figure 1. 


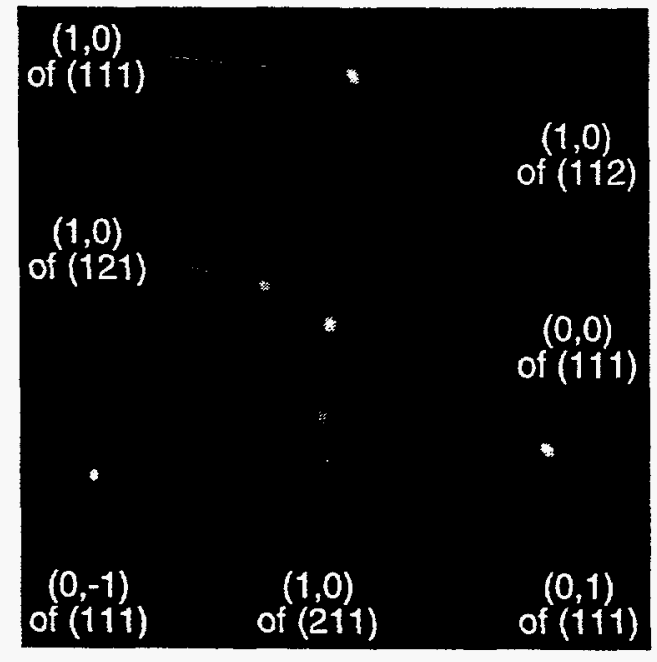

Figure 2. 


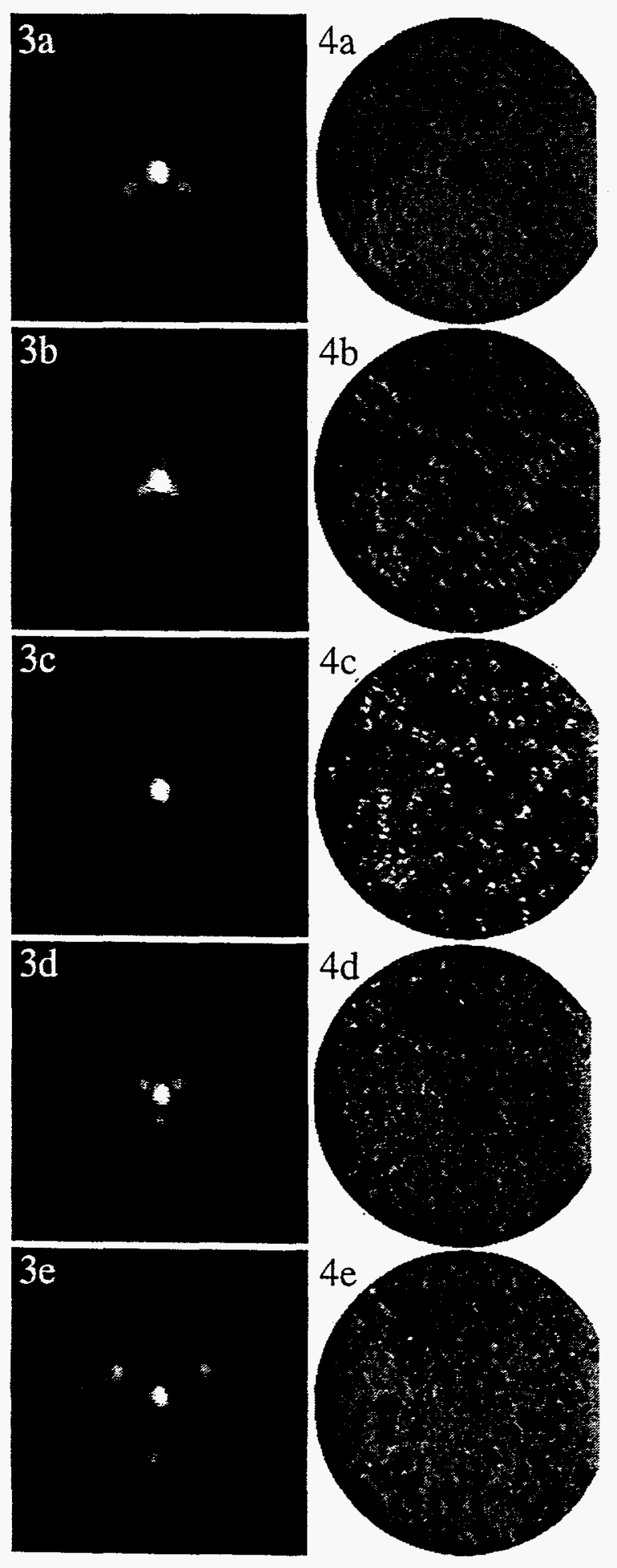

Figure 3 and 4 


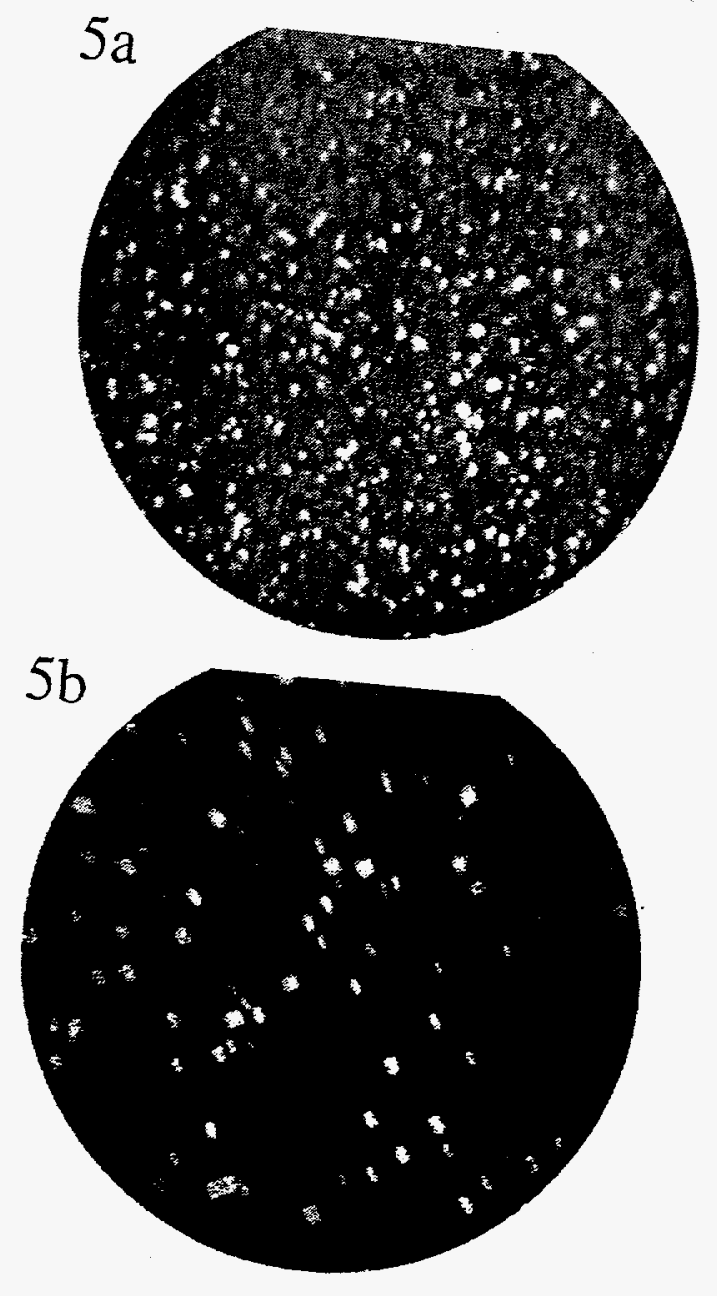

Figure 5. 

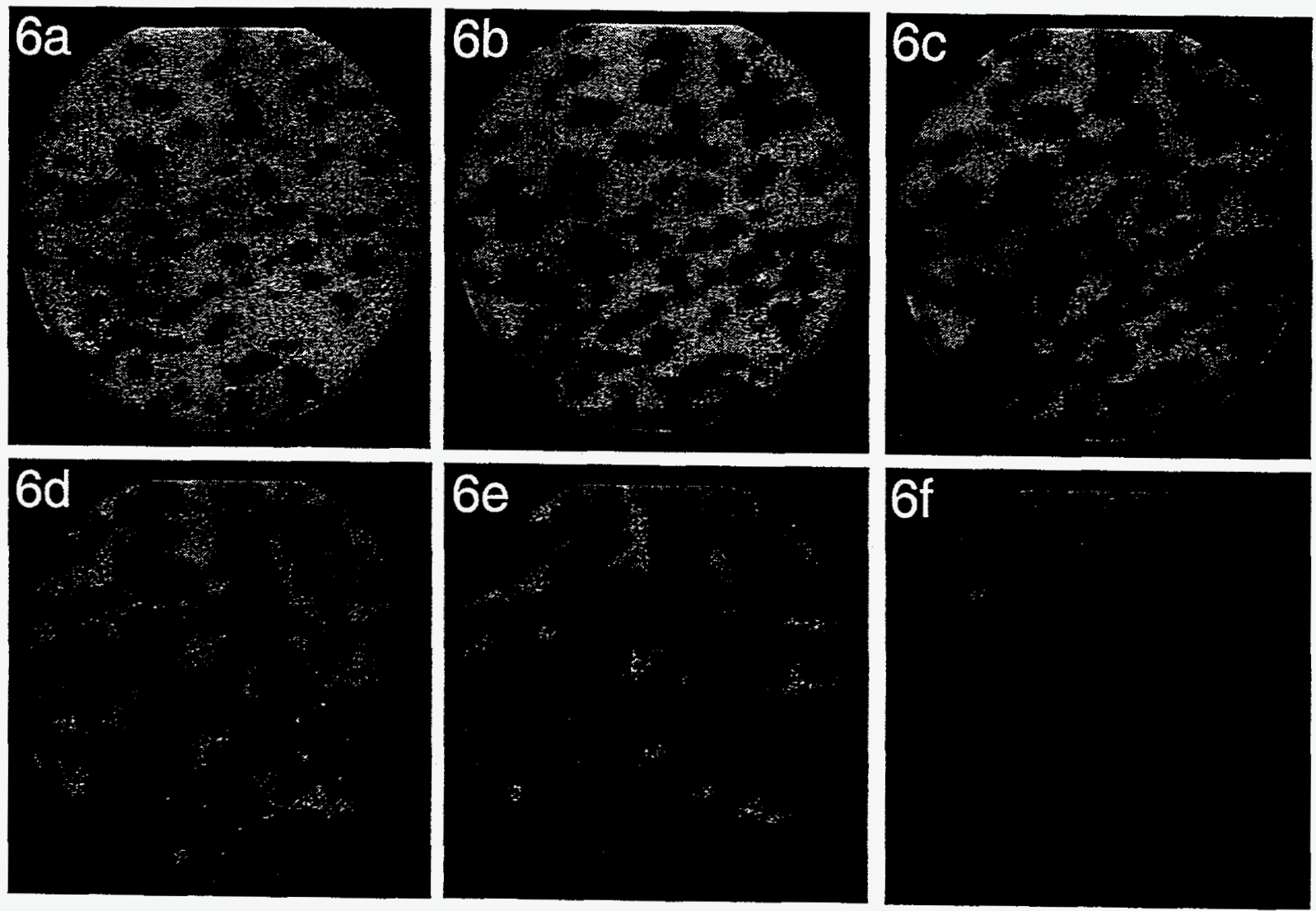

Figure 6. 